\title{
Celiac Plexus Block After Stereotactic Body Radiotherapy Improves Pain Relief in Locally Advanced Pancreatic Cancer
}

This article was published in the following Dove Press journal: Journal of Pain Research

Kai Ji'
Yue-Juan Shao'
Jian-Lei Hao'
Xian-Jiang Cheng'
Bing-Qing Guan'
Wei-Shuai Liu'
Lei Chen'
Xin Wang ${ }^{2}$
Yong-Chun Song ${ }^{2}$
Kun Wang'
Ping Wang (D ${ }^{2}$
'Department of Pain Relief, Tianjin
Medical University Cancer Institute and
Hospital, National Clinical Research
Center for Cancer, Key Laboratory of
Cancer Prevention and Therapy, Tianjin,
and Tianjin's Clinical Research Center for
Cancer, Tianjin, People's Republic of
China; 'Department of Radiotherapy,
Tianjin Medical University Cancer
Institute and Hospital, National Clinical
Research Center for Cancer, Key
Laboratory of Cancer Prevention and
Therapy, Tianjin, and Tianjin's Clinical
Research Center for Cancer, Tianjin,
People's Republic of China

People's Republic of China

\begin{abstract}
Purpose: This study evaluated the analgesic effect of stereotactic body radiotherapy (SBRT) in combination with celiac plexus block (CPB), relative to SBRT alone, in locally advanced pancreatic cancer (LAPC) patients.

Patients and Methods: We reviewed medical records of all patients with LAPC, who received SBRT between 1 January 2017 to 31 August 2019 at our center. The average numeric rating scale (NRS) of $\geq 3$ was used in all patients at admission. We recorded average and worst NRS in a 24-hour period, and daily narcotic doses before SBRT, followed by weekly for 1 month and monthly for 3 months.

Results: A total of 23 patients in the SBRT group and 12 under SBRT + CPB who met the inclusion criteria were enrolled. All patients in the SBRT $+\mathrm{CPB}$ group received CPB within 10 days after SBRT. Pain intensity and narcotic consumption were comparable in both groups at initial assessment. However, a significant decrease $(P<0.05)$ in average NRS was recorded in the SBRT $+\mathrm{CPB}$ group relative to SBRT at 2, 3 and 4 weeks after SBRT. A comparison of daily narcotic consumption with baseline values showed a significant decrease in the SBRT $+\mathrm{CPB}$ group at 3 and 4 weeks after SBRT $(P<0.05)$, while no significant differences were observed in the SBRT group.
\end{abstract}

Conclusion: CPB after SBRT appears to be an effective therapeutic option in patients with LAPC and warrants further evaluation with increased number of patients in prospective clinical trials.

Keywords: cancer pain, celiac plexus block, stereotactic body radiotherapy, locally advanced pancreatic cancer

\section{Introduction}

Locally advanced pancreatic cancer (LAPC) is a lethal disease that is associated with multiple debilitating symptoms and a one-year survival rate of between $38.7 \%$ and $49.1 \%{ }^{1}$ Approximately $80 \%$ of patients diagnosed with the condition experience pain. ${ }^{2}$ Of note, pancreatic cancer-related pain can be difficult to control, even with high doses of analgesics. ${ }^{3,4}$ Moreover, pain, mainly of moderate to severe intensity accompanied by other non-pain symptoms can severely decrease a patient's quality of life $(\mathrm{QoL}) .^{5}$ It is, therefore, a major goal to optimize the QoL by managing LAPC symptoms, especially by providing adequate pain control. $^{3}$

Stereotactic body radiotherapy (SBRT) is a highly accurate radiotherapeutic technique, which can precisely deliver a dose to a specific tumor site, while 
maximally protecting surrounding normal tissues. ${ }^{6}$ In the recent past, an increasing amount of studies have demonstrated the role of SBRT in relieving pain in LAPC patients. $^{7-10}$ On the other hand, celiac plexus block (CPB) is well established as an effective technique for controlling pain or reducing narcotic consumption in patients with pancreatic cancer. ${ }^{3,4,11}$ It involves the destruction and subsequent fibrosis of the neural tissue of celiac ganglia by percutaneous or intraoperative injection of alcohol.

Currently, the analgesic effect of CPB in patients treated with SBRT is unclear. A randomized double-blind controlled trial found that pain scores in patients who underwent chemotherapy and/or radiotherapy with CPB were not significantly different from the control group at 1 month, although the situation significantly improved at 3 months after randomization. ${ }^{4}$ However, details regarding the radiotherapy technique as well as intervals between CPB and radiotherapy used in the study were not provided. Theoretically, pain relief could occur after SBRT, mainly due to direct irradiation-mediated tumor cell destruction. On the other hand, the analgesic effect of CPB results from destruction and subsequent fibrosis of the neural tissue. Given the different analgesic mechanisms of SBRT and $\mathrm{CPB}$, we hypothesized that SBRT in combination with CPB could potentially be superior to SBRT alone for alleviation of pain in LAPC patients.

In this study, therefore, we retrospectively analyzed data from single-center to determine whether SBRT combined with CPB could be superior to SBRT alone for the palliation of pain in LAPC patients.

\section{Patients and Methods}

\section{Patients}

Medical records for all patients with LAPC, who received SBRT from 1 January 2017 to 31 August 2019 at our center, were reviewed. All enrolled patients met the following criteria: (1) pancreatic cancer was histologically proven with imaging concordance; (2) unresectable lesion confirmed by a multidisciplinary team (MDT); (3) occurrence of continuous or intermittent visceral pain described as deep, not superficial; (4) the numeric rating scale (NRS) of pain (average in the last 24 hours) $\geq 3$ on admission; (5) ability to tolerate SBRT and/or CPB; and (6) expected survival time of $\geq 3$ months. Exclusion criteria included: (1) prior conventional fractionation radiotherapy of primary tumors, (2) coexistence of other primary tumors or distant metastasis, and (3) an interval time longer than 10 days between SBRT and CPB. All procedures performed in studies involving human participants were in accordance with the ethical standards of the Institutional Review Board (IRB) of Tianjin Medical University Cancer Institute \& Hospital and with the 1975 Declaration of Helsinki and its later amendments or comparable ethical standards. Written informed consent was obtained from all individual participants included in the study.

\section{Stereotactic Body Radiotherapy}

Patients with LAPC were treated with CyberKnife ${ }^{\circledR}$ (Accuracy, Sunnyvale, CA, USA) at our center according to a previously described treatment plan. ${ }^{12}$ Briefly, a fiducial marker (gold and of $0.8 \mathrm{~mm}$ diameter) was implanted into the tumor, guided by B-ultrasound, computed tomography (CT), or endoscopic ultrasonography. A simulation CT scan was then performed after one week. In the multi-plan treatment planning system, the gross tumor volume (GTV) was defined as visible primary lesion on simulation $\mathrm{CT}$, while the planning target volume (PTV) was formed by including a $3 \mathrm{~mm}$ margin around the GTV. The Synchrony respiratory motiontracking system and a special Synchrony vest donned by patients were used in treatment delivery.

\section{The Celiac Plexus Block Technique}

To perform $\mathrm{CPB}$, a patient was placed in a prone position, after which a pillow was placed under the patient's abdomen to achieve a thoracolumbar kyphotic position and CT scan was performed at $5 \mathrm{~mm}$ intervals. Puncture sites were $3.5-4.5 \mathrm{~cm}$ from both sides of the inferior border level of the T12 spinous process. After administration of local infiltration anesthesia using $5-10 \mathrm{~mL}$ of $1 \%$ lidocaine, spinal needles (22G, $13 \mathrm{~cm}$ long) were bilaterally inserted through the previously anesthetized area. The puncture procedure was performed under CT-guidance to avoid traversal of critical structures, such as large vessels and viscera. Ultimately, needle tips were laid just anterior to the lateral border of the vertebral body and just behind the aorta and vena cava in the retrocrural space. Correct bilateral needle placement was confirmed with $\mathrm{CT}$ images following injection of $3 \mathrm{~mL}$ of radio-opaque agent iohexol (iodine $350 \mathrm{mg} / \mathrm{mL}$; Beijing Beilu Pharmaceutical Co., Ltd, China) through each needle. Then, $6 \mathrm{~mL}$ of $1 \%$ lidocaine was injected through each needle for diagnostic and prognostic nerve block. Subsequently, $10 \mathrm{~mL}$ volume of $95 \%$ alcohol was administered bilaterally (a total of $20 \mathrm{~mL}$ ). 


\section{Evaluation of Pain Relief}

Quantitative pain assessment was performed using a 10point NRS, where 0 denotes "no pain" and 10 denotes "unbearable pain".

\section{Follow-Up}

The follow-up schedule began from the time of SBRT. The last follow-up was 21st November 2019. Each patient was asked to answer questions regarding the average and worst NRS in a 24-hour period as well as daily narcotic dose one day before SBRT $\left(\mathrm{W}_{0}\right)$, weekly after SBRT $\left(\mathrm{W}_{1}, \mathrm{~W}_{2}, \mathrm{~W}_{3}, \mathrm{~W}_{4}\right)$ for 1 month, and monthly $\left(\mathrm{M}_{2}, \mathrm{M}_{3}\right)$ for 3 months. This information was collected through periodic visits to the clinic or via telephone. Patients who had inadequate pain relief after SBRT or $\mathrm{CPB}$ were treated by medication according to guidelines of the analgesic regimen described by the World Health Organization (WHO). After SBRT, patients received imaging evaluations every 2 months, with examinations performed immediately when symptoms were observed. The Common Terminology Criteria for Adverse Events (CTCAE) version 5.0 were used to evaluate treatment-associated complications.

\section{Statistical Analysis}

Continuous data were presented as mean with standard deviations or medians with range while categorical datasets were shown as frequencies (percentages). Various narcotics and routes of administration were adopted; therefore, the doses were standardized by converting the doses into oral morphine-equivalents (OME, $\mathrm{mg} / \mathrm{d}$ ) as follows: $100 \mathrm{mg}$ oral morphine $=33 \mathrm{mg}$ intravenous or subcutaneous morphine $=50 \mathrm{mg}$ oral oxycodone $=50 \mathrm{mg} / \mathrm{h}$ transdermal fentanyl. ${ }^{13}$ Comparisons among parameters pertaining to patient characteristics were performed by using Fisher's exact test and Student's $t$-test. A paired $t$-test or Wilcoxon signed-rank test was applied for comparison within the groups. The differences in NRS and OME consumption between two groups were determined using the Student's $t$-test or Mann-Whitney $U$-test, while those in proportions of patients who used narcotics were compared using Fisher's exact test. Statistical significance was defined as a $P$ value $\leq 0.05$ in 2-tailed tests.

\section{Results}

Overall, 35 patients with LAPC treated with SBRT were eligible for inclusion in our analysis. The SBRT group included 23 while that with SBRT $+\mathrm{CPB}$ had 12 patients. By the end of the follow-up, in the SBRT group, 1 patient at 2 months and another at 3 months after SBRT were lost to follow-up. As a result, a total of 22 and 21 patients in SBRT group were included in the statistical analysis at 2 and 3 months, respectively. In the SBRT $+\mathrm{CPB}$ group, all patients received CPB within 10 days after SBRT. The median time between SBRT and CPB was 7 days (range: 5-10 days). The median age was 60 years (44-80 years). The median time from diagnosis to start of SBRT was 1 month (0.25-8 months), while median tumor volume was $40.9 \mathrm{~mL}(6.7-109.2 \mathrm{~mL})$. In addition, we found a median dose of SBRT to be 40 Gy (35-45 Gy), and this was delivered in 5 fractions. Most of the patients (71.4\%) received gemcitabine-based (alone or in combination with nab-paclitaxel, S-1 or oxaliplatin) chemotherapy. We noted no significant differences in baseline patient and treatment characteristics between the 2 groups (Table 1).

Pain intensity was comparable across both groups at initial assessment. At all time periods examined, the average and worst NRS were significantly lower in both groups compared with baseline values $(P<0.05)$. However, there was a significant decrease $(P<0.05)$ in average NRS in the $\mathrm{SBRT}+\mathrm{CPB}$ group relative to the SBRT group at 2, 3 and 4 weeks after SBRT (Figure 1 and Table 2). Two months after SBRT, differences in average NRS between the 2 groups approached statistically significant $(P=0.061)$. The worst NRS in the SBRT $+\mathrm{CPB}$ group was significantly lower $(P<0.05)$ than that in the SBRT group at 3 and 4 weeks after SBRT (Table 2).

The daily OME consumption and the proportion of patients who used narcotics were comparable in both groups at initial assessment. Differences in daily OME consumption as well as proportions of patients who used narcotics between the SBRT and SBRT $+\mathrm{CPB}$ groups were not significant across all time periods examined. The SBRT + CPB group showed a significant decrease in OME consumption compared to baseline values at 3 and 4 weeks $(P<0.05)$, while these parameters remained unchanged under SBRT alone (Table 3).

In addition, 11 patients (47.8\%) in SBRT group compared to $8(66.7 \%)$ in SBRT $+\mathrm{CPB}$ experienced grade $1-2$ malaise. Furthermore, 10 patients (43.5) in the SBRT group compared to $6(50.0 \%)$ under $\mathrm{SBRT}+\mathrm{CPB}$ had grade 1-2 radiation-induced gastrointestinal toxicity, including anorexia, abdominal distension, nausea, vomiting, and diarrhea. No grade 3 or above gastrointestinal toxicity was observed in the patients under study. Orthostatic hypotension was occurred in one patient after 
Table I Baseline Patient and Treatment Characteristics

\begin{tabular}{|c|c|c|c|}
\hline & $\begin{array}{l}\text { SBRT } \\
\text { Group } \\
(n=23)\end{array}$ & $\begin{array}{l}\text { SBRT + CPB } \\
\text { Group } \\
(n=12)\end{array}$ & $P$ value \\
\hline $\begin{array}{l}\text { Gender } \\
\text { Male } \\
\text { Female }\end{array}$ & $\begin{array}{l}10(43.5) \\
13(56.5)\end{array}$ & $\begin{array}{l}5(41.7) \\
7(58.3)\end{array}$ & 1.000 \\
\hline $\begin{array}{l}\text { Age, years } \\
\text { Mean } \pm \text { SD } \\
\text { Median } \\
\text { Range }\end{array}$ & $\begin{array}{l}61.4 \pm 9.1 \\
64.0 \\
45.0-80.0\end{array}$ & $\begin{array}{l}59.8 \pm 8.6 \\
59.5 \\
44.0-76.0\end{array}$ & 0.609 \\
\hline $\begin{array}{l}\text { Site of pancreatic mass } \\
\text { Head/uncinate } \\
\text { Body/tail }\end{array}$ & $\begin{array}{l}16(59.6) \\
7(30.4)\end{array}$ & $\begin{array}{l}9(75.0) \\
3(25.0)\end{array}$ & 1.000 \\
\hline $\begin{array}{l}\text { Time since diagnosis, } \\
\text { months } \\
\text { Mean } \pm \text { SD } \\
\text { Median } \\
\text { Range }\end{array}$ & $\begin{array}{l}2.3 \pm 2.1 \\
2.0 \\
0.25-8.0\end{array}$ & $\begin{array}{l}1.8 \pm 1.7 \\
1.0 \\
0.5-6.0\end{array}$ & 0.543 \\
\hline $\begin{array}{l}\text { Systemic therapy } \\
\text { Induction ChT before } \\
\text { SBRT } \\
\text { Maintenance ChT after } \\
\text { SBRT } \\
\text { Both induction ChT and } \\
\text { maintenance ChT } \\
\text { No ChT received }\end{array}$ & $\begin{array}{l}9(39.1) \\
4(17.4) \\
3(13.0) \\
7(30.4)\end{array}$ & $\begin{array}{l}4(33.3) \\
3(25.0) \\
2(16.7) \\
3(25.0)\end{array}$ & 0.923 \\
\hline $\begin{array}{l}\text { Pre-SBRT CA I9-9 } \\
(\mathrm{U} / \mathrm{mL}) \\
\text { Mean } \pm \text { SD } \\
\text { Median } \\
\text { Range }\end{array}$ & $\begin{array}{l}234.5 \pm 284.6 \\
168.4 \\
7.1-1031.0\end{array}$ & $\begin{array}{l}291.9 \pm 270.6 \\
216.2 \\
12.7-836.5\end{array}$ & 0.569 \\
\hline $\begin{array}{l}\text { SBRT dose (Gy) } \\
\quad 40-45 \\
35-39\end{array}$ & $\begin{array}{l}18(78.3) \\
5(21.7)\end{array}$ & $\begin{array}{l}9(75.0) \\
3(25.0)\end{array}$ & 1.000 \\
\hline $\begin{array}{l}\text { Tumor volume }(\mathrm{mL}) \\
\text { Mean } \pm \mathrm{SD} \\
\text { Median } \\
\text { Range }\end{array}$ & $\begin{array}{l}43.7 \pm 25.0 \\
40.7 \\
6.7-109.2\end{array}$ & $\begin{array}{l}45.7 \pm 18.8 \\
45.6 \\
11.7-79.0\end{array}$ & 0.801 \\
\hline $\begin{array}{l}\text { Pain location } \\
\text { Abdominal } \\
\text { Back } \\
\text { Both }\end{array}$ & $\begin{array}{l}\text { II (47.8) } \\
7(30.4) \\
5(21.7)\end{array}$ & $\begin{array}{l}6(50.0) \\
3(25.0) \\
3(25.0)\end{array}$ & 1.000 \\
\hline $\begin{array}{l}\text { Pain temporal pattern } \\
\text { Intermittent } \\
\text { Constant }\end{array}$ & $\begin{array}{l}9(39.1) \\
14(60.9)\end{array}$ & $\begin{array}{l}3(25.0) \\
9(75.0)\end{array}$ & 0.476 \\
\hline
\end{tabular}

Note: Values are presented as $\mathrm{n}(\%)$ unless otherwise defined.

Abbreviations: SBRT, stereotactic body radiotherapy; CPB, celiac plexus block; ChT, chemotherapy; CA 19-9, carbohydrate antigen 19-9.

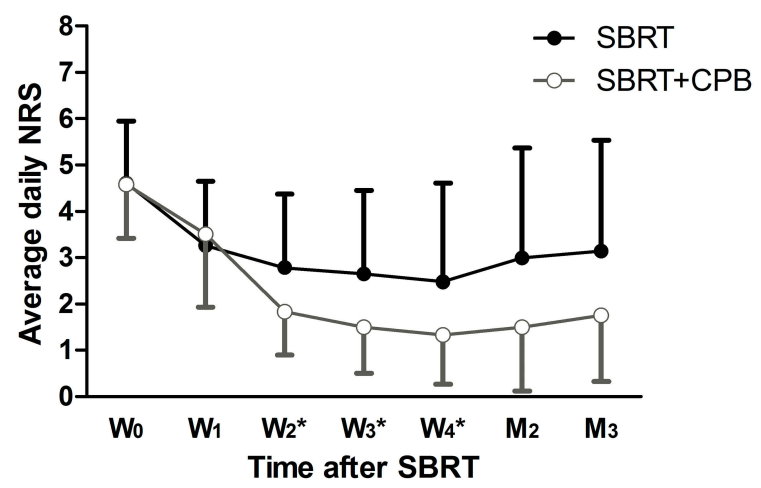

No. of

Patients

$\begin{array}{llllllll}\text { SBRT } & 23 & 23 & 23 & 23 & 23 & 22 & 21\end{array}$

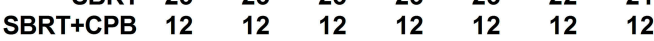

Figure I The average NRS from baseline through 3 months. *There are significant differences between two groups.

Abbreviations: NRS, numeric rating scale; SBRT, stereotactic body radiotherapy; CPB, celiac plexus block; $W_{0}$, one day before SBRT; $W_{1}, W_{2}, W_{3}, W_{4}$, weekly after SBRT; $M_{2}, M_{3}$, monthly after SBRT.

$\mathrm{CPB}$ and resolved within 24 hours with the administration of adequate amounts of intravenous fluids.

\section{Discussion}

In patients with pancreatic cancer, SBRT treatment relieves pain caused by tumor-induced ductal obstruction and pancreatic neuropathy by direct killing of tumor cells. Furthermore, CPB can interrupt nociceptive impulses originating from the pancreas through an irreversible chemical ablation of coeliac plexus. Theoretically, a better analgesic effect may be achieved by combining SBRT and CPB. Currently, to the best of our knowledge, none has explored the analgesic effect of SBRT in combination with CPB in LAPC patients. This study reveals that both treatments significantly improved pain relief, and the improvement was even more pronounced in combined CPB and SBRT treatment at 3 and 4 weeks after SBRT. In addition, a decrease in narcotic consumption was evident in the SBRT + CPB group at 3 and 4 weeks. However, this difference was lost later on. Although the actual reasons remain unclear, pain aggravation due to metastatic lesions and the consequent increase of narcotics might account for this.

When compared to conventionally fractionated radiotherapy, SBRT yields higher survival rates or a decrease in toxicity. ${ }^{14,15}$ Besides, the effect of SBRT in pain control in LAPC patients has been reported in some studies. Most 
Table 2 Pain Intensity for Both Groups

\begin{tabular}{|c|c|c|c|c|c|c|}
\hline \multirow[t]{2}{*}{ Time } & \multicolumn{3}{|l|}{ Average NRS } & \multicolumn{3}{|l|}{ Worst NRS } \\
\hline & SBRT Group & SBRT+CPB Group & $P$ value & SBRT Group & SBRT+CPB Group & $P$ value \\
\hline$W_{0}$ & $4.6 \pm 1.3$ & $4.6 \pm 1.2$ & 0.943 & $6.5 \pm 1.9$ & $6.8 \pm 1.4$ & 0.502 \\
\hline$w_{1}$ & $3.3 \pm 1.4$ & $3.5 \pm 1.6$ & 0.676 & $4.7 \pm 2.0$ & $5.7 \pm 2.0$ & 0.200 \\
\hline$W_{2}$ & $2.8 \pm 1.6$ & $1.8 \pm 0.9$ & $0.047^{\mathrm{a}}$ & $3.9 \pm 2.1$ & $3.1 \pm 1.2$ & 0.191 \\
\hline$W_{3}$ & $2.7 \pm 1.8$ & $1.5 \pm 1.0$ & $0.048^{\mathrm{a}}$ & $3.8 \pm 2.4$ & $2.6 \pm 1.1$ & $0.047^{\mathrm{a}}$ \\
\hline$W_{4}$ & $2.5 \pm 2.1$ & $1.3 \pm 1.1$ & $0.042^{\mathrm{a}}$ & $3.5 \pm 2.9$ & $1.8 \pm 1.4$ & $0.022^{\mathrm{a}}$ \\
\hline$M_{2}$ & $3.0 \pm 2.4$ & $1.5 \pm 1.4$ & 0.061 & $4.2 \pm 3.3$ & $2.7 \pm 1.7$ & 0.182 \\
\hline$M_{3}$ & $3.1 \pm 2.4$ & $1.8 \pm 1.4$ & 0.083 & $4.5 \pm 3.4$ & $3.0 \pm 2.0$ & 0.150 \\
\hline
\end{tabular}

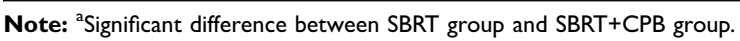

Abbreviations: SBRT, stereotactic body radiotherapy; CPB, celiac plexus block; $W_{0}$, one day before SBRT; $W_{1}, W_{2}, W_{3}, W_{4}$, weekly after SBRT; $M_{2}, M_{3}$, monthly after SBRT.

Table 3 Intra-Group Comparison of Daily OME Consumption for Both Groups

\begin{tabular}{|l|l|l|l|l|}
\hline Time & SBRT Group (No. of Patients) & $P$ value (vs $\mathbf{W}_{\mathbf{0}}$ ) & SBRT+CPB Group (No. of Patients) & $P$ value (vs $\left.\mathbf{W}_{\mathbf{0}}\right)$ \\
\hline$W_{0}$ & $57.4 \pm 71.7(15)$ & & $58.3 \pm 51.5(9)$ & \\
$W_{1}$ & $45.2 \pm 48.3(14)$ & 0.622 & $50.8 \pm 41.7(9)$ & 0.279 \\
$W_{2}$ & $49.1 \pm 63.3(13)$ & 0.858 & $40.0 \pm 40.2(7)$ & 0.121 \\
$W_{3}$ & $48.7 \pm 69.5(13)$ & 0.474 & $30.0 \pm 34.9(7)$ & $0.016^{\mathrm{a}}$ \\
$W_{4}$ & $49.6 \pm 84.0(11)$ & 0.627 & $25.8 \pm 33.7(6)$ & $0.007^{\mathrm{a}}$ \\
$M_{2}$ & $72.7 \pm 98.7(13)$ & 0.593 & $37.5 \pm 59.1(6)$ & 0.149 \\
$M_{3}$ & $98.1 \pm 108.2(12)$ & 0.173 & $54.2 \pm 76.8(7)$ & 0.827 \\
\hline
\end{tabular}

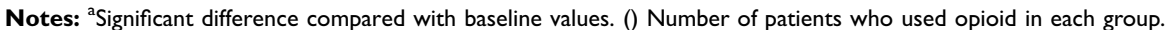

Abbreviations: OME, oral morphine-equivalents; $S B R T$, stereotactic body radiotherapy; CPB, celiac plexus block; $W_{0}$, one day before $S B R T ; W_{1}, W_{2}, W_{3}$, $W_{4}$, weekly after SBRT; $M_{2}, M_{3}$, monthly after SBRT.

studies reported pain relief after SBRT. In a Phase 2 study of gemcitabine and fractionated SBRT, patients with LAPC reported a significant improvement in pain relief 4 weeks after SBRT on the QLQ-PAN26 questionnaire. ${ }^{8}$ This is consistent with our study, where the lowest average and worst NRS occurred at 4 weeks after SBRT. In addition, we found a decrease in narcotic consumption with the improvement in pain relief. In a series of 25 patients with LAPC or metastatic disease, Su reported $50.0 \%$ and $15.0 \%$ suspension of analgesic administration and reduced analgesic consumption rates after SBRT, respectively. ${ }^{10}$ Seo treated 30 patients in a Phase 1 dose escalation study using a single SBRT fraction dose as a boost after conformal RT. Analgesic consumption was reduced in 10 of the 18 patients $(55.6 \%)$ after radiotherapy. ${ }^{16}$ In our study, however, 17 patients still used narcotics after 4 weeks of SBRT compared to the 24 patients before SBRT. This may be attributed to the fact that narcotics are required for the optimal analgesic effect after SBRT in some patients who did not initially use narcotics.

At least two good quality studies have shown strong evidence supporting that, in comparison with analgesic treatments alone, CPB provides an improvement in pain relief., ${ }^{3,4}$ The subgroup analysis of one of these studies suggested that early CPB was not effective in providing pain relief at 1 month, in patients who underwent chemotherapy and/or radiotherapy, ${ }^{4}$ which is inconsistent with the findings of our study. This inconsistency may be attributed to a longer time interval, from diagnosis to start of CPB (mean 2.4 months), in the current study compared to that of the previous study. Moreover, details about the radiotherapy technique used, dose, fractionation scheme and interval between CPB and radiotherapy were not provided in the aforementioned study.

Previous studies have suggested that pain is associated with poor prognosis in patients with pancreatic cancer. In a study analyzing of 136 patients who underwent palliative gastric bypass, Muller reported median survival times of 9.4, 7.6 and 3.5 months $(P=0.0017)$ for patients with "not daily", "daily" and "daily and strong" pain, respectively. ${ }^{17}$ Another study of 149 patients, who underwent pancreatic tumor resection, was classed into three groups based on their pain intensity and frequency, and measured survival as the time between surgery and cancer-specific death. ${ }^{18}$ The median survival times for patients with no pain, mild pain, and 
moderate to severe pain were $21.5,15.0$, and 10.0 months, respectively $(p=0.0015) .{ }^{18}$ Given the dismal prognosis of painful pancreatic cancer, any low-risk therapy that may relieve pain is likely to have an important role in patient management. In the current study, although SBRT could relieve pain in patients with LAPC, CPB after SBRT provided better pain relief and a decrease in narcotic consumption over a period of time, and as a consequence an improvement of QoL potentially.

Because of the inherent flaws in a retrospective study with a relatively small sample and short follow-up period, the statistical power is limited and confounding variables may exist. As a result, the advantage of CPB for relieving abdominal or back pain may not be consistently displayed. In addition, there could have been a possible selection bias due to a stronger desire by patients who underwent CPB after SBRT to control pain or reduce narcotic consumption. Moreover, we did not record non-opioid consumption, and this may contribute to either overestimation or underestimation of the analgesic effects. Despite the aforementioned limitations, given that few studies have explored the combined use of CPB and SBRT, we believe that our findings lay the foundation for future studies focusing on pain control in patients with LAPC.

\section{Conclusion}

The findings of this study show that, in comparison with SBRT alone, CPB after SBRT can provide better pain relief and reduce narcotic consumption over a period of time. We believe that these results should, at the very least, encourage further evaluations of the analgesic efficacy of CPB in combination with SBRT in LAPC patients during future prospective clinical trials.

\section{Disclosure}

The authors report no conflicts of interest in this work.

\section{References}

1. Amini A, BL J, Stumpf P, et al. Patterns of care for locally advanced pancreatic adenocarcinoma using the National Cancer Database. Pancreas. 2017;46(7):904-912. doi:10.1097/MPA.0000000000000876

2. Koulouris AI, Banim P, Hart AR. Pain in patients with pancreatic cancer: prevalence, mechanisms, management and future developments. Dig Dis Sci. 2017;62(4):861-870. doi:10.1007/s10620-017-4488-z

3. Wong GY, Schroeder DR, Carns PE, et al. Effect of neurolytic celiac plexus block on pain relief, quality of life, and survival in patients with unresectable pancreatic cancer: a randomized controlled trial. JAMA. 2004;291(9):1092-1099. doi:10.1001/jama.291.9.1092
4. Wyse JM, Carone M, Paquin SC, Usatii M, Sahai AV. Randomized, double-blind, controlled trial of early endoscopic ultrasound-guided celiac plexus neurolysis to prevent pain progression in patients with newly diagnosed, painful, inoperable pancreatic cancer. J Clin Oncol. 2011;29(26):3541-3546. doi:10.1200/JCO.2010.32.2750

5. Shao YJ, Ji K, Hao JL, et al. Nonpain symptom prevalence and intensity of inpatients with moderate to severe cancer pain in China. Am J Hosp Palliat Care. 2016;33(5):448-455. doi:10.1177/ 1049909114565109

6. Buwenge M, Cilla S, Guido A, et al. Individually optimized stereotactic radiotherapy for pancreatic head tumors: a planning feasibility study. Rep Pract Oncol Radiother. 2016;21(6):548-554. doi:10.1016/ j.rpor.2016.09.003

7. Buwenge M, Macchia G, Arcelli A, et al. Stereotactic radiotherapy of pancreatic cancer: a systematic review on pain relief. J Pain Res. 2018;11:2169-2178. doi:10.2147/JPR.S167994

8. Herman JM, Chang DT, Goodman KA, et al. Phase 2 multi-institutional trial evaluating gemcitabine and stereotactic body radiotherapy for patients with locally advanced unresectable pancreatic adenocarcinoma. Cancer. 2015;121(7):1128-1137. doi:10.1002/ cncr.29161

9. Rao AD, Sugar EA, Chang DT, et al. Patient-reported outcomes of a multicenter phase 2 study investigating gemcitabine and stereotactic body radiation therapy in locally advanced pancreatic cancer. Pract Radiat Oncol. 2016;6(6):417-424. doi:10.1016/j.prro.2016.05.005

10. Su TS, Liang P, Lu HZ, et al. Stereotactic body radiotherapy using CyberKnife for locally advanced unresectable and metastatic pancreatic cancer. World J Gastroenterol. 2015;21(26):8156-8162. doi:10.3748/wjg.v21.i26.8156

11. Mercadante S, Klepstad P, Kurita GP, Sjogren P, Giarratano A. Sympathetic blocks for visceral cancer pain management: a systematic review and EAPC recommendations. Crit Rev Oncol Hematol. 2015;96(3):577-583. doi:10.1016/j.critrevonc.2015.07.014

12. Song Y, Yuan Z, Li F, et al. Analysis of clinical efficacy of CyberKnife((R)) treatment for locally advanced pancreatic cancer. Onco Targets Ther. 2015;8:1427-1431. doi:10.2147/OTT. S81939

13. Svendsen K, Borchgrevink P, Fredheim O, Hamunen K, Mellbye A, Dale O. Choosing the unit of measurement counts: the use of oral morphine equivalents in studies of opioid consumption is a useful addition to defined daily doses. Palliat Med. 2011;25(7):725-732. doi:10.1177/0269216311398300

14. Park JJ, Hajj C, Reyngold M, et al. Stereotactic body radiation vs. intensity-modulated radiation for unresectable pancreatic cancer. Acta Oncol. 2017;56(12):1746-1753. doi:10.1080/0284186X.2017.134 2863

15. Zhong J, Patel K, Switchenko J, et al. Outcomes for patients with locally advanced pancreatic adenocarcinoma treated with stereotactic body radiation therapy versus conventionally fractionated radiation. Cancer. 2017;123(18):3486-3493. doi:10.1002/cncr.30706

16. Seo Y, Kim MS, Yoo S, et al. Stereotactic body radiation therapy boost in locally advanced pancreatic cancer. Int J Radiat Oncol Biol Phys. 2009;75(5):1456-1461. doi:10.1016/j.ijrobp.2009.01.042

17. Muller MW, Friess H, Koninger J, et al. Factors influencing survival after bypass procedures in patients with advanced pancreatic adenocarcinomas. Am J Surg. 2008;195(2):221-228. doi:10.1016/j. amjsurg.2007.02.026

18. Ceyhan GO, Bergmann F, Kadihasanoglu M, et al. Pancreatic neuropathy and neuropathic pain-a comprehensive pathomorphological study of 546 cases. Gastroenterology. 2009;136(1):177-186. doi:10. 1053/j.gastro.2008.09.029 


\section{Publish your work in this journal}

The Journal of Pain Research is an international, peer reviewed, open access, online journal that welcomes laboratory and clinical findings in the fields of pain research and the prevention and management of pain Original research, reviews, symposium reports, hypothesis formation and commentaries are all considered for publication. The manuscript

management system is completely online and includes a very quick and fair peer-review system, which is all easy to use. Visit http:// www.dovepress.com/testimonials.php to read real quotes from published authors. 